\section{Compliance with timolol treatment in glaucoma}

\begin{abstract}
Purpose To assess levels of compliance in elderly patients on timolol eyedrops for glaucoma.

Methods A postal questionnaire was sent from the general practitioner to 86 patients over 55 years of age on repeat prescriptions for timolol eyedrops. The questionnaire asked details about the duration of treatment, family history, the level of understanding of the disease and the importance of treatment, other regular medication, side-effects attributed to the drops and how often patients omitted their drops. A search of practice and local hospital dispensing data was carried out to assess how frequently monthly repeat prescriptions for timolol eyedrops were actually dispensed over a 12 month period. This allowed a total volume to be calculated for each patient.

Results Twenty-four per cent of patients admitted to omitting eyedrops either occasionally or frequently. Fifty-one per cent were found to have had insufficient drops dispensed to comply with treatment as prescribed. In non-compliant patients the mean period without drops was 85 days of the year, with a maximum of 165 days.

Conclusion Compliance with treatment is poor and patients underestimate their level of defaulting when questioned.
\end{abstract}

Key words Glaucoma, Timolol eyedrops, Compliance

A.P. Rotchford
Hlabisa Hospital
Private Bag $\times 5001$
Hlabisa
KwaZulu-Natal
South Africa
K.M. Murphy
The Surgery
School Lane
Alconbury
Huntingdon
Cambs PE17 5EQ, UK

A number of studies have used a patient interview or questionnaire to assess the degree of non-compliance with prescribed treatment in chronic glaucoma and a wide variety of other chronic medical disorders. ${ }^{18}$ However this approach is open to the criticism that it leads to under-reporting of defaulting. ${ }^{4-12}$ The purpose of this present study was to obtain a more objective assessment of the level of noncompliance and to determine whether noncompliers showed any characteristic features that made them identifiable.
ALAN P. ROTCHFORD,

KAREN M. MURPHY

\section{Methods \\ Patient sample}

All patients over the age of 55 years in three large Cambridgeshire dispensing practices who were on repeat prescription for timolol eye drops (Timoptol, MSD; $0.25 \%$ or $0.5 \%$ ) were included in the study. The 86 patients $(1.4 \%$ of the population over 55 years) had a mean age of 77 years and the male:female ratio was 39:47.

\section{Procedure}

A postal questionnaire was sent from the general practitioner to all 86 patients. The questionnaire asked details about the duration of treatment, family history, the level of understanding of the disease and the importance of treatment, other regular medication, side-effects attributed to the drops and how often patients omitted their drops.

A search of practice and local hospital dispensing data was carried out to assess how frequently and what volume of monthly repeat prescriptions for timolol eyedrops were actually dispensed over a 12 month period. The number of drops in the $5 \mathrm{ml}$ Ocumeter metered-dose unit was measured by emptying three bottles, one drop at a time. Bottles contained on average 150 drops $(149,150,150)$. This is in agreement with company data on this product (delivers $30 \pm 3 \mathrm{drops} / \mathrm{ml}$ under clinical conditions). Thus it was possible to calculate the total number of drops dispensed for each patient.

\section{Results}

Completed questionnaires were returned by all 86 patients. Twenty-one $(24 \%)$ of the patients admitted to omitting treatment either frequently or occasionally. There was no correlation between these subjects and their age, sex, family history, duration of disease, understanding of glaucoma and the potential effects of noncompliance, whether they were taking other regular medication, and side-effects. Patients who said they always took their drops without fail were significantly more likely to regard their drops as being 'vital' as opposed to 
'important' ( $\chi^{2}$ analysis, $p=0.025$ ). Although there were only 13 patients registered as blind or partially-sighted, which limits significance, every one of these patients replied that they never missed doses and considered taking their drops to be vital. Side-effects were reported by 37 (45\%) patients; 'stinging' was the commonest, occurring in 22 patients (26\%). The potentially serious nature of glaucoma was appreciated by the majority of patients, $71(83 \%)$ understanding that vision may deteriorate if glaucoma goes untreated.

Complete dispensing information was available for 55 patients. For the remainder the hospital dispensing records were incomplete. Twenty-eight of 55 (51\%) did not have sufficient timolol eyedrops dispensed to medicate as prescribed. The average shortfall was 85 days over a 12 month period. This assumes that no timolol was wasted and that patients always finished each bottle. If instead some patients discard bottles after 1 month then clearly the shortfall will be greater. Sixtynine per cent overall ( $72 \%$ of the non-compliance group) said this was their normal practice which, if true, means that the figure of $51 \%$ non-compliance represents a minimum estimate.

Compliance with treatment was compared with the results of the questionnaire survey. No significant correlation emerged between non-compliance and any particular group of subjects. However, there was a trend which did not reach statistical significance between poor compliance and registration as blind/partially-sighted, with only one of the eight registered patients showing evidence of poor compliance compared with $54 \%$ of nonregistered patients.

\section{Discussion}

Many studies using a variety of techniques have demonstrated that a substantial proportion of patients on long-term treatment for glaucoma fail to self-medicate as prescribed. Depending on the precise degree of failure that constitutes non-compliance, estimates range from $27 \%$ to $59 \%$ of patients. ${ }^{1-5}$ These figures are in line with defaulting rates in a wide variety of medical disorders in which the benefits to the patient of using the medication are not immediately apparent. ${ }^{6-8}$

Most studies carried out previously have used direct interviewing or a questionnaire to obtain information on compliance. However, patients aware that they are supposed to take their medication are likely to underreport defaulting when questioned..$^{9-12}$ Other

investigators have used eyedrop medication monitors to record actual usage more objectively, albeit over a short period. ${ }^{9,10,13}$ Our study attempts to side-step this problem by calculating how much timolol each patient had dispensed to them relative to their need based on the regimen prescribed over a long period. The result that $51 \%$ of patients had insufficient drops dispensed to them contrasts with patients' own perception of their compliance, $76 \%$ claiming never to miss doses. Amongst the $51 \%$ who had insufficient drops the mean shortfall amounts to 85 days per patient. This still represents an underestimate of the level of defaulting since, of course, obtaining the eyedrops is no guarantee of their correct usage.

The reasons why patients default from treatment remain unclear and doctors are 'poor at guessing which of their patients are compliant and which are not'. ${ }^{14}$ Our study supports the view that there is no readily identifiable type of patient in terms of demographic and social characteristics who is a non-complier. ${ }^{15}$

The presence of another chronic medical complaint besides glaucoma, ${ }^{3}$ knowledge of the disease and the importance of treatment, ${ }^{2,4}$ and more serious loss of sight ${ }^{4}$ have been variously found to correlate with better compliance. However, none of these is a universal finding. ${ }^{3,5,16}$ In this study patients demonstrated a high level of awareness that failure to treat glaucoma may result in a deterioration of vision (83\%) and $90 \%$ understood the reason for attending clinic.

There was no relationship between lack of knowledge and poor compliance. We did, however, find that patients who had been registered as blind or partiallysighted were more compliant and all said that they never forgot their eyedrops.

Side-effects are commonly reported with all topical treatments in glaucoma, occurring in up to $77 \%$ of patients. ${ }^{17}$ It is far from clear, however, that this is a major factor in determining non-compliance. ${ }^{1,2,17}$ This study supports the high level of adverse effects ascribed to timolol without affecting compliance.

Compliance has been shown to be higher with timolol than pilocarpine, but it is unclear as to whether this is a result of fewer side-effects or a more convenient regime. ${ }^{13} \mathrm{~A}$ consistent finding is that non-compliance is significantly higher in those using drops more than twice daily, ${ }^{1,2,18,19}$ in whom the midday dose is particularly likely to be omitted. ${ }^{2}$ This should be taken into account when assessing new therapies with shorter efficacy.

Compliance should never be assumed in patients who fail to respond to treatment. Non-compliant patients are at risk of being subjected to unnecessary multiple drug regimes with increasing adverse effects and worsening compliance or to filtration surgery. It remains the responsibility of the clinician to consider this possibility of non-compliance and as far as practicable to prescribe once- or twice-daily drops.

There is also some evidence that linking selfmedication with other routine activities, for example meal times, may help overcome forgetfulness. ${ }^{9}$ Intensive periods of education have been shown to increase compliance at least for a while, ${ }^{20}$ and every opportunity should be used to remind patients of the importance of their treatment.

In general practice the usual mechanism used for ensuring drug compliance is a 6 to 12 monthly medication review, at which the GP enquires about the medications taken. This information is then correlated with the computer records of numbers of tablets/bottles prescribed. However, in our experience glaucoma medication is often perceived very differently by GPs. They do not feel directly involved in the management of 
glaucoma in the way that they do for instance in diabetes and hypertension, and often have very little information from the hospital with which to reinforce compliance. As a consequence review and monitoring of long-term ophthalmic medications is not carried out in general practice.

If compliance is to be improved then shared care needs to be emphasised and GPs encouraged to be more involved in reinforcing patient compliance in the community. This might be achieved by establishing better communication links between the clinic and the GP. Alternatively patient-held prescription records updated by the prescribing physician would enable hospital staff to see how much medication is being collected as well as keeping GPs informed about any changes in medication.

\section{References}

1. Patel SC, Spaeth GL. Compliance in patients prescribed eyedrops for glaucoma. Ophthalmic Surg 1995;26:233-6.

2. Mackean JM, Elkington AR. Compliance with treatment of patients with chronic open angle glaucoma. $\mathrm{Br} \mathrm{J}$ Ophthalmol 1983;67:46.

3. Bloch S, Rosenthal AR, Friedman L, Caldarolla P. Patient compliance in glaucoma. Br J Ophthalmol 1977;61:531-4.

4. Spaeth GL. Visual loss in a glaucoma clinic. Invest Ophthalmol Vis Sci 1970;9:73-82.

5. Vincent PA. I'atients' viewpoint of glaucoma therapy. SightSaving Rev 1972;42:213-21.

6. Blackwell B. Drug therapy: patient compliance. N Engl J Med 1973:289:249-52.
7. Stimson GV. Obeying doctor's orders: a view from the other side. Soc Sci Med 1974;8:97-104.

8. Rudd P. Clinicians and patients with hypertension: unsettled issues about compliance. Am Heart J 1995;130:572-9.

9. Granstrom P-A. Glaucoma patients not compliant with their drug therapy: clinical and behavioural aspects. $\mathrm{Br} \mathrm{J}$ Ophthalmol 1982;66:464-70.

10. Kass MA, Meltzer DW, Gordon M, Cooper D, Goldberg J. Compliance with topical pilocarpine treatment. Am J Ophthalmol 1986;101:515-23.

11. Gordis L, Markowitz M. The inaccuracy in using interviews to estimate patient reliability in taking medication at home. Med Care 1969;7:49-54.

12. Norrell SE. Accuracy of patient interviews and estimates by clinical staff in determining medication compliance. Soc Sci Med 1981;15:57-61.

13. Kass MA, Gordon M, Morley R, Meltzer D, Goldberg J. Compliance with topical timolol treatment. Am J Ophthalmol 1987;103:188-93.

14. Yager J. Cognitive aspects of illness. In: Pasnam RO, editor. Consultation-liaison psychiatry. New York: Grune and Stratton, 1975.

15. Porter AMW. Drug defaulting in general practice. BMJ 1969;1:218-22.

16. Sackett DL, Gibson ES, it al. Randomised clinical trial of strategies for improving medication compliance in primary hypertension. Lancet 1975;I:1205-7.

17. Lehto I. Side effects of topical treatment in pigmentary glaucoma. Acta Ophthalmol (Copenh) 1992;70:225-7.

18. Davidson SI, Akingbehin T. Compliance in ophthalmology. Trans Ophthalmol Vis Sci 1970;7:73-82.

19. Gurwitz HG, Glynn RJ, Monane M, Everitt MD, Gilden D, Smith N, Avorn J. Treatment for glaucoma: adherence by the elderly. Am J Public Health 1993;83:711-6.

20. Norell SE. Improving medication compliance: a randomised clinical trial. BMJ 1979;II:1031-3. 